\title{
A Fast Initial Alignment Method for SINS Used Adaptive Sample Size Unscented Particle Filter
}

\author{
Jiaxu Yang *, Xueguang yuan, Yangan Zhang \\ State Key Laboratory of Information Photonics and Optical Communication, \\ Beijing University of Posts and Telecommunications, \\ Beijing 100876, China \\ *E-Mail: sun3ly@163.com;Tel./Fax:: +86-010-6119-8081
}

\begin{abstract}
A fast initial alignment method used Adaptive sample size unscented particle filter (AUPF) for SINS is proposed in this paper. As the SINS initial alignment with large misalignment angle is a typical nonlinear and non-Gaussian filtering problem, the unscented particle filter (UPF) is widely used in existing alignment technologies. UPF suffers from the inherent drawback of costly calculation, even though it is considered as the optimal nonlinear estimate method up to now. The new method overcomes the huge computational burden by embedding KLD-Sampling method into the re-sampling procedure of the standard UPF, so that the number of particles can ensure to be least dynamically. In addition, a quaternion-based nonlinear error model is established to not only describe the propagation of errors, but also play an important role in the implementation of filters. To verify the efficiency of the proposed method, the turntable test and computer simulations are conducted. The results of the simulations demonstrate that the new method can effectively reduce the calculation and improve the real-time performance.
\end{abstract}

Keywords-strapdown inertial navigation system; initial alignment; quaternion-based error model; adaptive sample size unscented particle filter; KLD-Sampling method

\section{INTRODUCTION}

Initial alignment is one of the critical issues of Strapdown Inertial Navigation System (SINS) [1,2]. As the first work stage of SINS, the initial alignment error which caused by inertial sensors and alignment method error largely affected the performance of the system. The initial azimuth accuracy is one of the main factors which influence the navigation accuracy of SINS [3]. Thus, it is important to estimate initial attitude and reduce misalignment angles. The basic objective of initial alignment is to determine the initial strapdown attitude matrix, which is a transformation matrix between body frame and navigation frame. For most navigation systems, the initial azimuth is often large by suffering from much external interference, the linear error model based on small misalignment angle is unsuitable to express the error propagation of navigation system [4].The traditional linear Kalman filter can't meet the precision demand, so the research of initial alignment with large misalignment angle is very important [5].

Since SINS is nonlinear and the sensor noise obeys a non-Gaussian distribution, a nonlinear and non-Gaussian error model should be adopted to get a better estimation precision. In addition, an appropriate estimation method is in great request when developed a SINS, especially for the nonlinear and non-Gaussian system [6]. As the development of parallel computation technology and decrease of save cost, particle filter frame is widely used in navigation systems. The Unscented Particle Filter (UPF) improves the traditional particle filter by using unscented transformation to get a better importance sampling density. Thus, it combines the merits of both unscented transformation and particle filtering for state estimation [7]. The UPF was considered as one of the most effective state estimation method for nonlinear and non-Gaussian system. However, UPF had the inherent drawback of costly calculation that will impact the performance of the system. Therefore how to reasonably reduce the computation becomes a critical problem in UPF.

This paper presents a new method by adopting the quaternion concept to SINS to overcome the shortcomings of the linear SINS error model. A quaternion-based matrix representation is derived for describing the attitude of SINS[8]. Using this attitude matrix representation, quaternion-based nonlinear error model is established for the system, which contains high-order error items to accurately describe the nonlinear characteristics and the variation of attitude errors. Since the quaternion-based state is strongly nonlinear and non-Gaussian, an adaptive sample size unscented particle filtering (AUPF) method is developed based on the quaternion nonlinear models for fusion of multiple sensor data [9]. The new method overcomes the drawback of standard UPF by using KLD-Sampling to determine the number of particles. The KLD-Sampling chose the number of particles online to reduce the drawback of computational burden. Turntable test and comparison analysis of computer simulations have been conducted to comprehensively evaluate the performance of the proposed method for SINS.

The outline of the reminder of this paper is as follows. An improved initial alignment method by choosing the number of particles online is first introduced in Section II . In Section III the turntable test and computer simulations for the proposed method are carried out. Section IV is the conclusion.

\section{QUATERNION-BASED ADAPTIVE SAMPLE SIZE UPF METHOD WITH KLD-SAMPLING}

\section{A. Quaternion-based Nonlinear SINS Error Model}

Navigation frame (n-frame): The establishment of the kinematic model is E-N-U (East-North-Up) geographic coordinate system. 
Body frame (b-frame): The orthogonal reference frame aligned with inertial measurement unit (IMU) axes.

Inertial frame (i-frame): The frame in inertial space.

$C_{b}^{n}$ : The direction cosine matrix (DCM) represents the rotation from frame $\mathrm{b}$ to frame $\mathrm{n}$.

$w_{n b}^{b}:$ The rotation rate between $\mathrm{n}$-frame and $\mathrm{b}$-frame resolved in b-frame.

SINS: Strapdown inertial navigation system.

KLD: Kullback-Leibler distance.

The process of initial alignment is as shown in Figure 1.

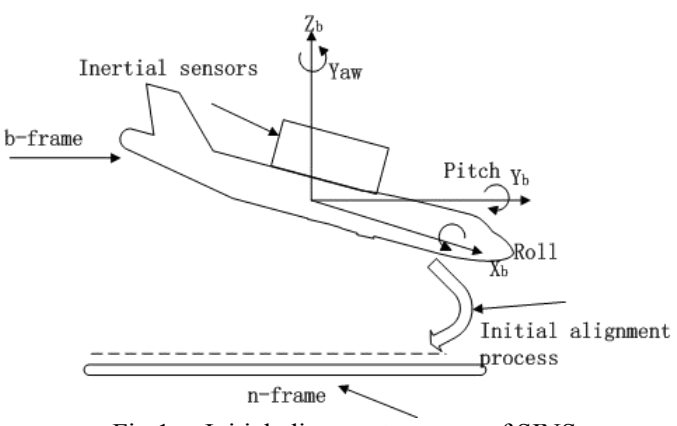

Fig.1. Initial alignment process of SINS

\section{B. Quaternion-based Nonlinear SINS Error Model}

Due to various error sources, the attitude information of SINS is not accurate. The error growth significantly affects the performance of SINS. Therefore, establishment of a SINS error model is an important issue. As quaternion is the most concise and efficient mathematical concept for description of the general displacement of a rigid body and can provide better accuracy and avoid singularity with less computational time, a quaternion-based nonlinear error model is established in this paper. Assume that $\varphi_{b}$ and $\varphi_{n}$ are the projection of a physical vector along the axes of the body frame and the navigation frame respectively, the vector $\varphi_{b}$ is usually the output of a body fixed sensor. The relationship between them is:

$$
\varphi_{n}=C_{b}^{n} \varphi_{b}
$$

Quaternion $Q_{b}^{n}=\left[q_{0}, q_{1}, q_{2}, q_{3}\right]^{T}=\left[q_{0}, q_{1-3}^{T}\right]^{T} \quad$ can describe this relationship, where $\mathrm{q}=\left[\mathrm{q}_{1-3}\right]^{\mathrm{T}}$ and $\mathrm{q}_{0}$ are scalar and vector part of the attitude quaternion respectively. Utilize quaternion to represent $C_{b}^{n}$ is as follow:

$$
\begin{aligned}
& \mathrm{C}\left(Q_{b}^{n}\right)= \\
& {\left[\begin{array}{ccc}
q_{0}^{2}+q_{1}^{2}-q_{2}^{2}-q_{3}^{2} & 2\left(q_{1} q_{2}-q_{0} q_{3}\right) & 2\left(q_{1} q_{3}+q_{0} q_{2}\right) \\
2\left(q_{1} q_{2}+q_{0} q_{3}\right) & q_{0}^{2}-q_{1}^{2}+q_{2}^{2}-q_{3}^{2} & 2\left(q_{2} q_{3}-q_{0} q_{1}\right) \\
2\left(q_{1} q_{3}-q_{0} q_{2}\right) & 2\left(q_{2} q_{3}+q_{0} q_{1}\right) & q_{0}^{2}-q_{1}^{2}-q_{2}^{2}+q_{3}^{2}
\end{array}\right]}
\end{aligned}
$$

AssumeQ is the real attitude quaternion and $\tilde{Q}$ is the quaternion obtained through calculation. There is:

$$
\widetilde{\mathrm{Q}}=\delta \mathrm{Q}^{-1} \otimes \mathrm{Q}
$$

$\delta \mathrm{Q}=\mathrm{Q} \otimes \widetilde{\mathrm{Q}}^{-1}$

Where, is attitude error caused by. The quaternion attitude error equation is derived in [10],

$\delta \dot{\mathrm{Q}}=-\frac{1}{2} \delta \widetilde{\mathrm{w}}_{\mathrm{ib}}^{\mathrm{n}} \otimes \delta \mathrm{Q}+\frac{1}{2} \delta \mathrm{Q} \otimes \widetilde{\mathrm{w}}_{\mathrm{in}}^{\mathrm{n}}-\frac{1}{2} \mathrm{w}_{\mathrm{in}}^{\mathrm{n}} \otimes \delta \mathrm{Q}$
Where $w_{r b}^{r}$ is the rotation rate between $r$-frame and b-frame resolved in $r$-frame, and the superscript " $\sim$ " denotes the measured value.

\section{The KLD-Sampling}

The Kullback-Leibler distance (KLD) provides a method to measure the fit of the distribution represented by weighted particles. It is used to determine the minimum number of particles needed to maintain the approximation quality in the sampling process, namely the KLD-sampling. This paper applies the KLD measure in the resampling process of the traditional UPF.

The KLD is used to represent the approximate error between the two probability distribution $\mathrm{p}$ and $\mathrm{q}$, as

$\mathrm{K}(\mathrm{p}, \mathrm{q})=\sum_{\mathrm{x}} \mathrm{p}(\mathrm{x}) \log \frac{\mathrm{p}(\mathrm{x})}{\mathrm{q}(\mathrm{x})}$

Assume that there is a discrete distribution with $\mathrm{k}$ different subspaces. The vector $\mathrm{X}=\left\{\mathrm{X}_{1}, \mathrm{X}_{2}, \ldots, \mathrm{X}_{\mathrm{k}}\right\}$ describes the number of sampling particles from different subspaces. The vector $\mathrm{P}=\left\{\mathrm{p}_{1}, \mathrm{p}_{2}, \ldots, \mathrm{p}_{\mathrm{k}}\right\}_{\text {describes the true probability of different }}$ subspaces. And the maximum likelihood estimate is described as $\widehat{\mathrm{P}}=\mathrm{X} / \mathrm{N}$. According to theory of probability, the K-L distance can be less than the threshold only while $\mathrm{N}$ is large enough. Then the approximate error between estimate and true probability density will be least.

The required number $\mathrm{N}$ of samples can be approximately obtained through the Wilson-Hilferty transform, which yields, $\mathrm{N}=\frac{\mathrm{k}-1}{2 \varepsilon}\left(1-\frac{2}{9(\mathrm{k}-1)}+\sqrt{\frac{2}{9(\mathrm{k}-1)}} \mathrm{z}_{1-\delta}\right)^{3}$

Where is upper quartile of the standard normal $\mathrm{N}(0,1)$ distribution. The method choose a small number of particles if the density was focused on a small subspace of the state space, and it chose a large number of samples if the state uncertainty was high.

\section{The Adaptive Sample Size Unscented Particle Filter Method}

The basic idea of the particle filter (PF) is to use a set of random samples particles $\left\{\mathrm{x}_{\mathrm{k}}^{\mathrm{i}}, \mathrm{i}=1,2 \ldots \mathrm{N}\right\}$ and related importance weights $\left\{w_{\mathrm{k}}^{\mathrm{i}}, \mathrm{i}=1,2 \ldots \mathrm{N}\right\}$ to approximate the posterior probability function and the estimation. With the number of the sample points increasing, Monte Carlo simulation property is approximately equal to the posterior probability density function. However in standard PF, the importance sampling density function did not use the latest measurement information. There will be a large error between the proposed distribution and the true posteriori probability density distribution. The unscented particle filter (UPF) method is an improved PF with the same recurrence structure. As unscented Kalman filter (UKF) is a high accurate three order filter, it is used to get better importance density functions. The UPF method uses UKF to generate a proposal distribution, which can integrate latest observation information to get the posterior probability distribution and is more in line with the true state. As the UPF takes advantage of the good features of both UKF and PF, and avoids their limitations, it is considered as the approximately optimal estimation method for nonlinear 
and non-Gaussian system. But there are still challenges for UPF such as particle impoverishment and the fading out of particle diversity in the sampling process. Aim at these problems, re-sampling method is used to resolve the degeneracy phenomenon, which is considered as the key problem of UPF. Although the accuracy of UPF can meet the requirement of the practical requirements, it suffers from great drawback of costly calculation caused by using a fixed size of particles. Since the complexity of the posterior distribution varies drastically over time, the sample size should adjust online according to the requirements of the system. The new method proposed in this paper solved this problem by embedding the KLD-Sampling method into the re-sampling step of the standard UPF. The UPF method is described in[11] in detail. Based on the above analysis, a flow chart of the proposed AUPF method is shown in Figure 2, in which the threshold got from KLD method is derived in equation (7).

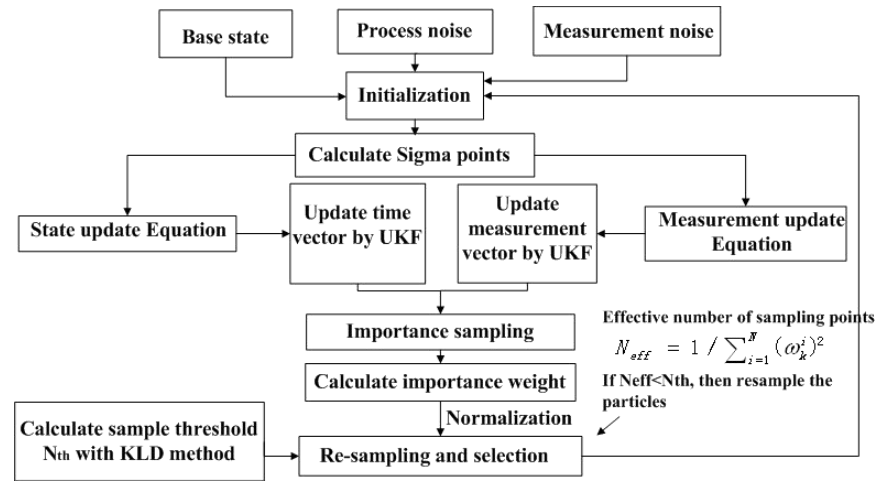

Fig.2. The flow chart of AUPF method

The proposed method chooses the number of samples online, when the system will resample the particles, duplicates particles with large weights and suppresses particles with low weights. Therefore, the number of particles is promised to be as least as possible in the new method.

\section{EXPERIMENT ANALYSIS AND DISCUSSIONS}

\section{A. Construction of Experiment}

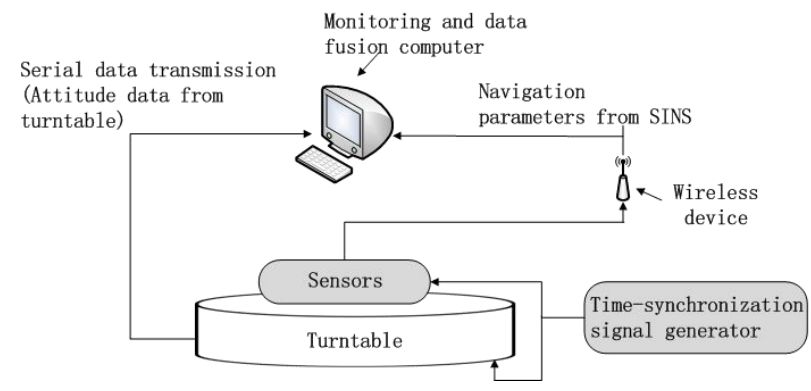

Fig. 3. Construction of turntable test

The turntable test is carried out in this study to verify the efficiency of the proposed method, and the construction of the experiment is shown in Figure 3. The rate controlling error and the angle measuring error of the turntable are ignored in this paper. The sensors are consisted of an accelerometer and a gyroscope, which provides three-axis gyroscopes and accelerometers to the computer. The precisions of the accelerometer and the gyroscope are listed in Table I. The time-synchronization signal module controls the startup of the whole system and promises the synchronization between turntable and sensors. The monitoring and data fusion computer performs simulations with data from sensors and turntable. The computer is also the display module, which will show the estimation results at the same time of computing.

The experimental system worked as follows: 1) start the time-synchronization signal generator, 2) then the current attitude information of the turntable will be sent to the computer via serial port, 3 ) the current data from sensors will be sent to the computer at the same time via wireless device for initial alignment solution. With the data from turntable and sensors stored in the computer, evaluation can be executed on the initial alignment results, where the result obtained from monitoring and data fusion computer is the current estimated attitude, and the turntable data is the real attitude. And the matching error caused by transmission time-delay should be compensated before evaluation.

TABLE I. THE PRECISIONS OF SENSORS

\begin{tabular}{|c|c|c|}
\hline Sensors & Constant error & Random error \\
\hline Gyroscope () & 0.1 & 0.3 \\
\hline Accelerometer $(\mathrm{mg})$ & 5 & 1 \\
\hline
\end{tabular}

\section{B. Experiment results analysis}

In order to verify the efficiency of the proposed method, we choose the traditional UPF method for comparison in the computer simulation part. The simulation of the attitude estimation executed on the computer with MATLAB tool. The simulations of the two methods are based on the quaternion nonlinear error model established in 2.2. The test lasts for 100 seconds. Alignment errors of roll and yaw and pitch are shown in Figure 4(a-c) respectively. Black solid lines in the Figure 4 are the estimation errors of the AUPF method, while Blue dotted dash lines are the estimation errors of the UPF method. The curves show the estimate accuracy and convergence performance of UPF and AUPF clearly.

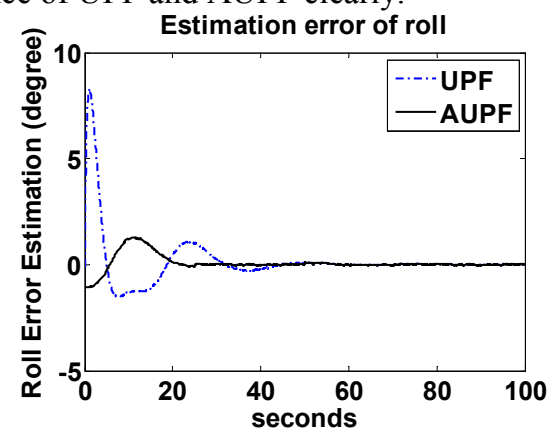

(a) 


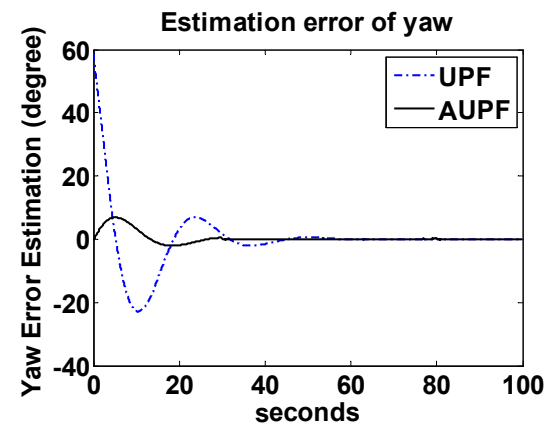

(b)

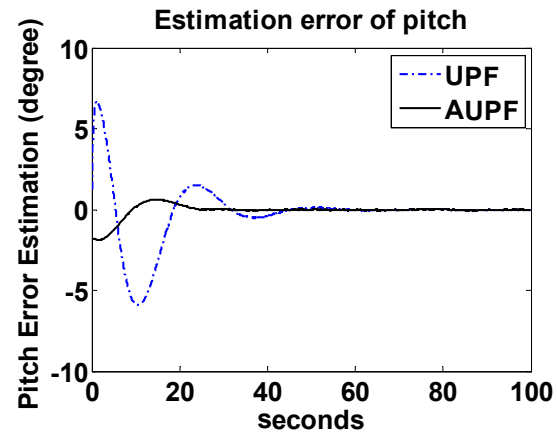

(c)

Fig.4. Estimation error comparison of UPF and AUPF

From the simulation results we can conclude that under the same initial conditions, UPF method will cost more convergence time. This is because of the number of particles in UPF method is fixed, so that unnecessary computation will happen in some steps. The number of particles used in some operating steps of the two methods is listed in Table II .

TABLE II. THE PRECISIONS OF SENSORS.

\begin{tabular}{|c|c|c|c|c|c|c|c|}
\hline $\boldsymbol{R U N S T E P}$ & 1 & 10 & 20 & 30 & 40 & 50 & 60 \\
\hline $\boldsymbol{U P F}$ & 300 & 300 & 300 & 300 & 300 & 300 & 300 \\
\hline $\boldsymbol{A U P F}$ & 300 & 300 & 247 & 176 & 321 & 290 & 116 \\
\hline
\end{tabular}

TABLE III. THE PRECISIONS OF SENSORS.

\begin{tabular}{|c|c|c|c|c|}
\hline $\boldsymbol{R M S E}$ & & & & $\begin{array}{c}\text { Running } \\
\text { time/(s) }\end{array}$ \\
\hline UPF & 2.32 & 2.13 & 4.56 & 213 \\
\hline AUPF & 2.12 & 1.95 & 4.03 & 135 \\
\hline
\end{tabular}

Figure 4 shows that the attitude estimate errors of UPF method converge within $50 \mathrm{~s}$, and the estimate errors of AUPF method converge within 20s, the yaw estimate error is bigger. The convergence time of the proposed method is less than half of the standard UPF method. Running 100 steps with the two methods respectively, the estimated state root-mean-square error and the consumption of time are indicated in Table 3.

From the comparison above, we can find that the two methods have approximately the same estimation accuracy, but the proposed method has better convergence in time than the standard UPF. The estimation results of the AUPF method converge to the true value in a short time with high accuracy, thus it provides better real-time performance and is more suitable for nonlinear and non-Gaussian state estimation. To sum up, the proposed initial alignment method can realize the initial alignment faster in experimental environment.

\section{CONCLUSIONS}

In this paper, an adaptive sample size unscented particle filter based on quaternion error model for initial alignment of SINS is proposed. In the method, we first established the quaternion-based nonlinear error model, which can not only improve estimate accuracy but also avoid singularity with less computational time. And then, the KLD-sampling method was embedded into standard UPF frame, to reduce the unnecessary computation caused by the fixed number of particles used in standard method. The improved method chose sample number online according to the requirements of the system. At last, a turntable test and computer simulations are carried out to verify the efficiency of the new method compare to standard UPF.

Simulation results demonstrate that the AUPF behaves better in real-time property than the standard UPF for initial alignment. What is more, the alignment accuracy of the two methods is extremely close to the theoretical value. Hence the proposed method is much more effective and suitable in SINS initial alignment. While more tests should be executed and the robustness of the proposed method should be studied before it can be widely used in engineering application.

\section{REFERENCES}

[1] X. Liu, X. Xu, Y. Liu, L. Wang, A fast and high-accuracy transfer alignment method between M/S INS for ship based on iterative calculation, J. Measurement. 51 (2014) 297-309.

[2] Y. Zhang, Y. Huang, Z. Wu, N. Li, Moving State Marine SINS Initial Alignment Based on High Degree CKF, J. Mathematical Problems in Engineering. 2014 (2014) 1-8.

[3] Y. Lu, X. Cheng, Random misalignment and lever arm vector online estimation in shipborne aircraft transfer alignment,J. Measurement. 47 (2014) 756-764.

[4] S. Gao, W. Wei, Y. Zhong, Z. Feng, Rapid alignment method based on local observability analysis for strapdown inertial navigation system, .Acta Astronautica . 94(2014) 790-798.

[5] J. Ali, M. Ushaq, A consistent and robust Kalman filter design for in-motion alignment of inertial navigation system, J. Measurement. 42(2009) 577-582.

[6] H. Dai, S. Dai, Y. Cong, G. Zhao, G. Wu, Rapid transfer alignment of laser SINS using quaternion based angular measurement, J. Optik-International Journal for Light and Electron Optics. 124(2013) 4364-4368.

[7] D.J. Jwo, C.H. Tseng, J.C. Liu, H.D. Lee, Unscented Kalman filtering for single camera based motion and shape estimation, J. Sensors. 11( 2011) 7437-7454.

[8] Q. Li, Y. Ben, J. Yang, Coarse alignment for Fiber Optic Gyro SINS with external velocity aid, J. Optik - International Journal for Light and Electron Optics. 125 (2014) 4241-4245.

[9] J. Ali, M.R. Ullah Baig Mirza, Initial orientation of inertial navigation system realized through nonlinear modeling and filtering, $\mathrm{J}$. Measurement. 44(2011) 793-801.

[10] L. Ma, K. Wang, M. Shao, Initial alignment on moving base using GPS measurements to construct new vectors, J. Measurement. 46 (2013) 2405-2410.

[11] B. Wang, X. Xiao, Y. Xia, M. Fu, Unscented Particle Filtering for Estimation of Shipboard Deformation Based on Inertial Measurement Units, J. Sensors. 13(2013) 15656-15672. 